\title{
Evaluating research co-production: Protocol for the Research Quality Plus for Co-Production (RQ+ 4 Co- Pro) Framework
}

Robert K.D. McLean ( $\nabla$ rmclean@idrc.ca )

International Development Research Centre https://orcid.org/0000-0001-8084-4817

Fred Carden

Using Evidence Inc.

lan D. Graham

University of Ottawa

Alice B. Aiken

Dalhousie University

Rebecca Armstrong

National Disability Insurance Agency

Judy Bray

Canadian Cancer Society

Christine E. Cassidy

Dalhousie University

Olivia Daub

Ottawa Hospital Research Institute

Erica Di Ruggiero

University of Toronto

Leslie A. Fierro

McGill University

Michelle Gagnon

Public Health Agency of Canada

Alison M. Hutchinson

Deakin University

Roman Kislov

Manchester Metropolitan University

Anita Kothari

Western University

Sara Kreindler

University of Manitoba

Chris McCutcheon 
Ottawa Hospital Research Institute

Jessica Reszel

University of Ottawa

Gayle Scarrow

MSFHR: Michael Smith Foundation for Health Research

\section{Study protocol}

Keywords:

Posted Date: February 2nd, 2022

DOI: https://doi.org/10.21203/rs.3.rs-1217799/v1

License: (c) (1) This work is licensed under a Creative Commons Attribution 4.0 International License. Read Full License

Version of Record: A version of this preprint was published at Implementation Science Communications on March 14th, 2022. See the published version at https://doi.org/10.1186/s43058-022-00265-7. 


\section{Abstract}

\section{Background}

Research co-production is an umbrella term used to describe research users and researchers working together to generate knowledge. Research co-production is used to create knowledge that is relevant to current challenges and to increase uptake of that knowledge into practice, programs, products, and/or policy. Yet, rigorous theories and methods to assess the quality of co-production are limited. Here we describe a framework for assessing the quality of research co-production - Research Quality Plus for CoProduction ( $\mathrm{RQ}+4 \mathrm{Co}-\mathrm{Pro})$ - and outline our field test of this approach.

\section{Methods}

Using a co-production approach, we aim to field test the relevance and utility of the RQ+ 4 Co-Pro framework. To do so we will recruit participants who have led research co-production projects from the international Integrated Knowledge Translation Research Network. We aim to sample 16 to 20 coproduction project leads, assign these participants to dyadic groups (8 to 10 dyads), train each participant in the RQ+ 4 Co-Pro framework using deliberative workshops and oversee a simulation assessment exercise using RQ +4 Co-Pro within dyadic groups. To study this experience, we use a qualitative design to collect participant demographic information and project demographic information, and will use in-depth semi-structured interviews to collect data related to the experience each participant has using the RQ+ 4 Co-Pro framework.

\section{Discussion}

This study will yield knowledge about a new way to assess research co-production. Specifically, it will address the relevance and utility of using RQ +4 Co-Pro, a framework that includes context as an inseparable component of research, identifies dimensions of quality matched to the aims of coproduction, and applies a systematic and transferable evaluative method for reaching conclusions. This is a needed area of innovation for research co-production to reach its full potential. The findings may benefit co-producers interested in understanding the quality of their work, but also other stewards of research co-production. Accordingly, we undertake this study as a co-production team representing multiple perspectives from across the research enterprise, such as: funders, journal editors, university administrators, and government and health organization leaders.

\section{Contributions To The Literature}

- Research co-production is a strategy used to produce scientific and societal benefits. Co-production is used to create research that is more relevant to knowledge users and to increase uptake of research into practice, programs, products, and policy. Yet, rigorous theories and methods to assess the quality of co-production are limited. 
- Adapted from the validated Research Quality Plus (RQ+) approach, Research Quality Plus for CoProduction Research ( $R Q+4$ Co-Pro) presents an adaptable and modular framework for evaluating co-production quality that addresses the context of the research, its scientific rigour, its legitimacy, and how well it is positioned for use.

- This study is the first RQ+ 4 Co-Pro application. It will field test the utility of the framework for evaluating research co-production projects, using a sample of completed projects from an international research network: the Integrated Knowledge Translation Research Network (IKTRN).

- A field-tested co-production specific evaluation approach will contribute to the critical development of high-quality research co-production as a means of knowledge generation and application.

\section{Background}

Research co-production shows great promise for connecting science to societal problems. Research coproduction can be rigorous, ethical [1-7] and serve as a vehicle for generating and translating scientific findings into action [8]. Research on implementation science and scaling science [9] demonstrates that the use of rigorous research designs is only one consideration when implementing and scaling innovations - context, user/beneficiary perspectives and systems matter just as much. The active involvement of users (those who may move research findings in action) and beneficiaries (those who may be affected) can be a crucial predictor of success $[8,9,10,11]$.

Research co-production comes in many forms and under many different names. Among others, community academic partnership [11]; community based participatory research [12]; co-creation [13]; and integrated knowledge translation [14-16]. A research study involving experts from a range of five research co-production traditions [17] found that the definitions and motivations of each type of co-production research were very similar. While there are many different names, engaging the users of research in the research process is a common goal. Therefore, we anticipate the results of this study to hold potential beyond the immediate sample. See Table 1 for general definitions of selected co-production traditions.

\section{The need for better evaluation approaches for co-production}

There is growing dissatisfaction with the approaches available for assessing the quality of research coproduction. Traditional approaches to research quality assessment do not take into account engagement with knowledge users and, as such, do not address a key factor in the hypothesis behind research coproduction - that engagement of knowledge users makes a difference in the uptake and use of the evidence that research produces $[8,10]$.

Table 2 outlines the predominant forms of research evaluation - as classified and further discussed in Chapter 4.3 of Research Coproduction in Healthcare [25] - and describes how these approaches can undervalue research co-production.

\section{Objectives of the RQ+ 4 Co-Pro field test}


The purpose of this study is to field test the relevance and utility of an adapted research quality evaluation approach, that was first developed and validated by the International Development Research Centre. This approach, called Research Quality Plus (RQ+) $[29,30]$ has previously been used to assess applied and use-oriented research. For a full explication, see McLean et al. 2022 [29]. With this study we will test whether $\mathrm{RQ}+$ can be adapted for assessing the quality of co-production research[1]. This prototype adaptation is called the Research Quality Plus for Co-Production, or RQ+ 4 Co-Pro, framework (25). See Figure 1 below for key definitions of RQ+ and RQ+ 4 Co-Pro.

Two research questions guide the field test:

1. Is the $\mathrm{RQ}+4$ Co-Pro framework relevant for the evaluation of research co-production?

2. Is the RQ+ 4 Co-Pro framework useful for the evaluation of research co-production?

\section{The Research Quality Plus for Co-Production (RQ+ 4 Co-Pro) framework}

The adaptation of $\mathrm{RQ}+$ into the $\mathrm{RQ}+4$ Co-Pro framework is illustrated in Figure 2. $\mathrm{RQ}+4$ Co-Pro was first proposed by authors of this paper following their experience designing and using the initial RQ+ framework at IDRC, doing research evaluations internationally, and doing research co-production (25). This is a prototype rendition. The study described in this manuscript aims to field test the protype. In Appendix I the fully detailed RQ+ 4 Co-Pro framework template is provided for the interested reader; it includes the definitions of each framework component and the associated evaluative rubrics. Appendix II provides a crosswalk of the components of the $R Q+$ framework with the components of the $R Q+4$ Co-Pro framework.

The RQ+ 4 Co-Pro framework embraces the three tenets of the RQ+ Approach. These are: 1) context matters, 2) quality is multi-dimensional, and 3) assessments should be empirical and systematic. These are modified from the RQ+ framework to reflect the particularities of co-production research. Here we provide a description of how each tenet was tailored, and then introduce an all-of-framework infographic (Figure 2) to show how the three tenets fit together.

\section{Contextual Factors}

Research always occurs in a context. Research is affected by and affects the socio-economic, historical, cultural, and political contexts as well as the geographic setting.[1] Attention to context is particularly important in the evaluation of the quality of research co-production [31]. We identify three contextual factors that can be monitored and categorized in a co-production evaluation. The goal in examining contextual factors is to gather information that can help to understand and navigate the enabling environment for co-production research. Understanding context is important to research design, management, and funding decisions as it helps clarify potential risks and opportunities and might also help with the development of strategies to capitalize on these and monitor progress. The contextual factors are not intended to affect the ratings of research quality dimensions or sub-dimensions, nor is any 
rating of a contextual factor necessarily 'better' than another. Rather, they help to provide a deeper understanding of the enabling environment.

The three $R Q+4$ Co-Pro contextual factors are: 1) Knowledge Use Environment, 2) Research Environment, and 3) Capacities for Co-Production. In the International Development Research Centre's current RQ+ Framework there are five contextual factors. Three are closely aligned to those here, given some tailoring to match co-production specifically. The additional two contextual factors, Data Environment and Maturity of the Research Field are not included in $R Q+4$ Co-Pro as they present less immediate alignment with the aims of co-production. The decision to reduce the number and tailor the contextual factors for $\mathrm{RQ}+4$ Co-Pro was the result of consultations between authors of this paper, and their shared experiences with co-production and co-production evaluations [25]. With this field test, we will further examine the relevance of these three contextual factors and determine the need to modify, exclude or include new elements on grounds of relevance and/or utility (see research questions above). Appendix II provides a crosswalk of the RQ+ contextual factors vis-à-vis the $R Q+4$ Co-Pro contextual factors.

\section{Quality Dimensions and Sub-Dimensions}

To assess co-production quality, we identify three dimensions and eight sub-dimensions. These are summarized in Figure 2 and presented in detail in Appendix I. Appendix II crosswalks these dimensions and sub-dimensions with those of the RQ+ framework.

As with all research, Scientific Rigour is central to co-production research and therefore comprises the first dimension. Two sub-dimensions are identified under Scientific Rigour. 1.1. the Protocol which addresses issues of study design, and 1.2. Methodological Integrity which assesses the rigour and integrity of the application of the study design. Research Legitimacy is the second dimension of $R Q+4 \mathrm{Co}$-Pro. There are four sub-dimensions to Legitimacy that assess the conformity, or fidelity of the research to the operating environment and relevance of the results for intended beneficiaries. These are: 2.1 Inclusion of Local Knowledge and Ways of Knowing, 2.2. Trust, Power and Mutually Beneficial Partnerships, 2.3. Intersectionality, and 2.4. Attention to Potentially Negative Consequences. The third and final dimension is Positioning for Use. It assesses the utility of the co-production research through examining 3.1. Relevance or how well the work is aligned to a current problem, and 3.2. Openness and Actionability which addresses accessibility and usefulness of the research findings.

In the $R Q+4$ Co-Pro Framework, all dimensions are interrelated and should not be considered as variables that are independent of each other. They are disaggregated to promote a deeper understanding of the multiple dimensions of research quality - ultimately, they must be considered as a set. We assign equal weight to the dimensions and sub-dimensions; others may choose to prioritize or highlight some subdimensions over others in any assessment they design.

\section{Empirical and Systematic Appraisal}


Column 3 in Figure 2 outlines the scale to be used for measurement. RQ+ 4 Co-Pro users apply a rubric for measurement which ensures transparency in the results and promotes a systematic approach across all the research that is being assessed. A combination of qualitative explanations and quantitative measures of sub-dimensions should be used to reach conclusions about the quality of the co-production research. In the following sections, we outline how empirical evidence will be gathered in our field test.

Table 3 below outlines how RQ+ 4 Co-Pro addresses some key recommendations from studies on research co-production approaches.

\section{Study Design}

This field test will use a multiple method qualitative design. It will include training of participants, standardized data collection using desk-based templates, and follow-up qualitative interviews with both the assessors and those whose projects have been assessed. As well, it will include a consultative process with the project team for revising $\mathrm{RQ}+4$ Co-Pro based on the outcomes of the field test $[35,36]$.

The study will take a research co-production approach. To do so, the study is being undertaken as a partnership between researchers and knowledge users. All activities and responsibilities will be shared, yet, five team members (authors: RKDM, FC, IDG, AK, CM) are primarily responsible for study design and execution. Thirteen team members (authors: ABA, RA, JB, CEC, OD, EDR, LAF, MG, AMH, RK, SK, JR, GS) hold primary responsibility for identifying knowledge uptake and use opportunities. These 'knowledge user' team members represent critical stewardship roles for research co-production broadly, including: funders, university administrators/leaders, research evaluation specialists, journal editors, co-production trainees, research managers, and co-production scientists. By working together to field test RQ+ 4 Co-Pro we hope to spark reasoned and appropriate uptake of the framework into settings where current coproduction evaluation techniques demand revision and innovation.

The field test will be implemented in four phases, which comprise eight steps. Figure 3 presents an illustration of the complete research life cycle.

\section{PHASE 1 - STUDY PREPARATION}

\section{Sample recruitment and participant consent}

Researchers in the Integrated Knowledge Translation Research Network (IKTRN) will be invited to submit projects for assessment and volunteer to assess another project. IKTRN is a network of researchers with an interest in both using and carrying out research on integrated knowledge translation. IKTRN is funded by a multi-year grant from the Canadian Institutes of Health Research [19]. The sample will be a convenience sample. This sample will be drawn from researchers with IKT research experience (members of the IKTRN) and who have recently completed an IKT project (have an IKT case published in the IKTRN 
casebook series). This invitation will be delivered by email from the study PI to eligible members of the IKTRN, until the desired sample size of 16 projects is reached, with a maximum of 20 projects. Enrolled participants will be arranged in dyads based on research topic familiarity for the assessment.

The sample range (16-20 projects) is based on two factors. The first is viability given resource requirements of past experiences using the $\mathrm{RQ}+$ approach, and our own study timelines and resources for this project. The second is the anticipated saturation point of qualitative data collected in the field test [37].

\subsection{Eligibility criteria}

We will consider eligibility at two levels: (1) the IKT research project and (2) the individuals participating in our study, as described below in tables $4 \& 5$ respectively. The research team will gather informed written consent from all participants.

\section{2. $R Q+4$ Co-Pro framework training}

Participants will receive training in $\mathrm{RQ}+4$ Co-Pro. Training will be provided by the core research team, with the aim to introduce the RQ+ 4 Co-Pro framework, the definitions and meaning of its components (contextual factors, quality dimensions and subdimensions, evaluative rubrics) and systematize the approach to its use by participants. The 2-hour training will be completed prior to the initiation of all data collection.

\section{PHASE 2 - DATA COLLECTION}

Data collection will involve four steps: (1) completion of a participant socio-demographic form, (2) completion of a project information form, (3) dyadic RQ + 4 Co-Pro assessments, and (4) participating in an interview with the research team on the strengths and limitations of the RQ+ 4 Co-Pro framework.

\section{Step 1 - Participant socio-demographic form}

All participants will be sent a link to an online socio-demographic form. This form will collect information on participant demographics and their experience/background developing and/or delivering IKT research projects. We will ask that participants complete this form prior to taking part in the training session. (5 minutes)

\section{Step 2 - Project information form}

All participants will be sent a link to a project information form. This form will be pre-populated by the study team as much as possible to profile the project included in the field test. The study participant will verify and complete any missing information on the project profile prepared by the study team. (10 minutes) 


\section{Step 3 - Dyadic project assessment exercise}

Each participant in the paired dyad will provide the other, who will serve as assessor, with publicly available documentation on the project they will be assessing (inter alia publications, manuscripts, reports, briefs, blogs, etc). These assessors (study participants in dyads [38]) will review this material to gain an understanding of the project context, as well as its strengths and weaknesses (1-2 hours). Next, the assessors within each dyad will engage in an assessment interview about their projects using the $R Q+4$ Co-Pro training and the field test template (see Annex 1) provided by the research team. Assessment interviews may be done in one virtual call or split in two as the two determine. It is estimated they will last 60 minutes per project. The field test template will be used by the assessors for recording results of the assessment during the interview.

\section{Step 4 - Research interview with RQ+ 4 Co-Pro study team}

On completion of the dyadic assessments, members of the research team (RKDM, FC) will interview the assessors (study participants) individually using a semi-structured interview guide to elicit their views as both assessor and assessed on the utility and relevance of using RQ+ 4 Co-Pro to assess the quality of IKT research [36]. The interviews will be completed by phone or video conference, depending on participant preference. Interviews will take approximately 60 minutes.

\section{PHASE 3 - ANALYSIS AND REVISION}

\section{Data Analysis}

Data analysis will be conducted for each data source independently (Participant demographic forms, Project information forms, Interviews with study participants), and triangulation will be conducted across the independent lines of evidence for congruence as well as instances of discordance.

\section{Step 1 - Participant demographic form analysis}

Frequencies will be generated for all closed-ended questions. Responses to open-ended questions will be analyzed for common and disparate themes using content analysis. Analysis will provide an overview of participants backgrounds and experiences brought to the field test.

\section{Step 2 - Project information form analysis}

The project profile forms will be analysed using content analysis to provide an overview of the nature of the projects included in the field test.

\section{Step 3 - Interview analysis}


Qualitative interview data collection and analysis will occur simultaneously so that identified themes can be incorporated into future interviews. Interviews will be audio recorded with permission of the interviewee. Where permission for transcription or recording are not granted the interview notes will be sent to the interviewee for review.

We will use thematic analysis [39] to identify patterns in the interview data. We will use an inductive or data-driven approach, without using a pre-existing coding frame. The coding will be modified based on new findings and in collaboration amongst interviewers. The first two interviews will be coded by two researchers independently and the results compared. Differences will be discussed to ensure agreement on a common approach for the remaining interviews. If agreement is not achieved between the two researchers, a third researcher will arbitrate opposing views and provide a third opinion to reach majority decision if consensus is not achieved.

\section{Step 4 - Triangulation and analysis}

As a final step in data analysis, we will look for similarities and differences of note in the study data by comparing findings across the lines of evidence. We will conduct triangulation by data source and by data collection method. Data will be considered in triangulation by using identified codes and themes to compare data. For example, we may cross tabulate all projects with a timeline of more than four years (as identified in the project information form), by perspectives around the importance, or lack-thereof, of using contextual factors in the RQ +4 Co-Pro framework. This is a hypothetical example. Triangulation will be driven by identified themes in the data.

\section{Revision of the $R Q+4$ Co-Pro Framework}

Based on the findings of the research, we will revise the prototype version of the RQ+ 4 Co-Pro framework. To facilitate this revision, the research team will host a meeting of all team members (including our knowledge users) to review and discuss preliminary research results and how these may induce the desire for change to the framework or its components. The reasons for changing a framework component will relate to the two research questions driving the study: relevance of the framework components and utility of the framework components and its application. Following team iteration, we will prepare any required revisions, and represent the revised framework to the study participants for review/member checking.

\section{PHASE 4 - RESULTS SHARING}

The final version of the RQ+ 4 Co-Pro framework will be published in a study findings report. This report will be submitted to an open access peer-reviewed journal for external assessment by co-production specialists. Uptake and use strategies will be developed by/amongst knowledge user perspectives represented on our co-production team.

\section{Discussion}


This study will yield knowledge about a new way to assess research co-production. Specifically, it will address the relevance and utility of using RQ+ 4 Co-Pro, a framework that includes context as an inseparable component of research, identifies dimensions of quality matched to the aims of coproduction, and applies a systematic and transferable evaluative method for reaching conclusions. As we have argued in this paper (see Table 1), evaluation is a needed area of innovation for research coproduction to reach its full potential. As we have presented (see Table 3), we are not alone in raising this call.

\section{Limitations}

There are limitations with our study design and study methods. The first is while we propose that the approach should apply to all research co-production approaches, we have limited our sample to IKT projects as a specific sub-domain of co-production which may limit generalization to other partnered research approaches. Second, our sample of IKTRN projects will prioritize experiences of the global North, as IKTRN membership is largely comprised of members from Canada, Australia, and the United Kingdom. Third, we further limited our sample to completed projects and so the study will not test the potential use of $\mathrm{RQ}+4 \mathrm{Co}$-Pro at the design and implementation stages of co-production projects. Fourth, given the evaluands in our field test are research projects, generalizability to other evaluands such as organizations, project portfolios, or grant applications should be tempered. Fifth, the approach to applying the framework will focus on discussion with principal investigators and documentary analysis. In future uses of the framework, users may wish to be more holistic and include more data sources, for example interviews with end users (however, if the framework is not considered useful to researchers, it will likely be problematic for knowledge users.) At the same time, other applications may go into less depth than we do in this field test, for example using a checklist for project design or application review. Using our design, we cannot be certain our field test experience will generalize to these other potential uses of the framework. Sixth, this field test is limited to health research projects although we recognize that co-production is an approach used in multiple domains of science. Finally, all study participants are part of the same network; this may risk a more positive assessments of each other's projects due to social bias. However, the goal of the research is to assess the relevance and utility of the framework, not to draw a final conclusion about the quality of co-production research endeavors sampled.

\section{Looking ahead}

We expect that our findings will add to the existing options for assessing co-production research that may benefit researchers but also other stewards of research co-production. Accordingly, we undertake this study as a co-production team with varied experiences and constituents we currently represent. Some potential uses may include funders interested in new ways to select, encourage and/or evaluate coproduction, including at different phases of the research life cycle. It may also give journal editors a higher level of comfort with the quality of research co-production they publish. Research institutions, such as universities or think tanks, may benefit from assessing the quality of co-production they do using a framework tailored to their values, objectives, and context. In the final research report of this RQ+ $4 \mathrm{Co}-\mathrm{Pro}$ 
field test, a section discussing users and uses of $\mathrm{RQ}+4$ Co-Pro will be elaborated. We will also tailor outputs and use strategies to the identified needs of knowledge users within our team. These efforts may not appear in peer-reviewed journals or other scholarly publication formats, but instead as use-oriented outputs and activities.

\section{Declarations}

Ethics approval and consent to participate

Approval has been obtained from the Research Ethics Board of the Ottawa Health Science Network (OHSN-REB 20210642-01H). All participants will sign consent to participate forms and are free to leave the study at any point, for any reason. Confidentiality is assured for study participants.

\section{Consent for publication}

Not applicable.

\section{Availability of data and materials}

No study data has been collected yet. Upon study completion, please contact the corresponding author for more information.

\section{Competing interests}

\section{IDG is the scientific director of the IKTRN, AK is the deputy director of the IKTRN, $\mathrm{CM}$ is the manager of the IKTRN, JR is the research coordinator of the IKTRN. AMH is protocols editor at Implementation Science.}

\section{Funding}

Funding for this study is provided by the Canadian Institutes of Health Research Foundation Grant (FDN \#143237) entitled, Moving knowledge into action for more effective practice, programs and policy: A research program focusing on integrated knowledge translation.

\section{Authors' contributions}

RKDM, FC, IDG conceptualized the research and study protocol. RKDM and FC drafted the manuscript and facilitated all reviews and revisions following co-author feedback. All authors (RKDM, FC, IDG, ABA, $\mathrm{RA}, J \mathrm{~J}, \mathrm{CEC}, \mathrm{OD}, \mathrm{ED}, \mathrm{LAF}, \mathrm{MG}, \mathrm{AMH}, \mathrm{RK}, \mathrm{AK}, \mathrm{SK}, \mathrm{CM}, \mathrm{JR}, \mathrm{GS}$ ) made contributions to the conception and design of the work and reviewed, contributed to, and approved the submitted manuscript.

\section{Acknowledgements}

We acknowledge the support of the members of the Integrated Knowledge Translation Research Network and its members who have encouraged this study. 


\section{References}

1. Lavery, JV. Building an Evidence base for Stakeholder Engagement. Science. 2018; 6361 (6402): 55455.

2. Eccles MP, Mittman BS. Welcome to implementation science. Implement Sci. 2006;1(1):1. doi:10.1186/1748-5908-1-1

3. Barwick M, Dubrowski R, Petricca K. Knowledge Translation: The Rise of Implementation.; 2020. https://ktdrr.org/products/kt-implementation/KT-Implementation-508.pdf

4. Davis R, D'Lima D. Building capacity in dissemination and implementation science: a systematic review of the academic literature on teaching and training initiatives. Implement Sci. 2020;15(1). doi:10.1186/s13012-020-01051-6

5. Harries S. Bridging policy and delivery with knowledge: the case for intervention. In: Records Management and Knowledge Mobilisation. Elsevier; 2012:145-168. doi:10.1016/b978-1-84334-653$1.50007-\mathrm{x}$

6. Ivankova N V., Creswell JW, Stick SL. Using Mixed-Methods Sequential Explanatory Design: From Theory to Practice. Field methods. 2006;18(1):3-20. doi:10.1177/1525822X05282260

7. Duncan s, Oliver s. Editorial: Motivations for Engagement. Science for All 2017; 1 (2). https://doi.org/10.18546/RFA.01.2.01.

8. Canadian Institutes of Health Research (2013). Evaluation of ClHR's Knowledge Translation Funding Program. ClHR, Ottawa. [online]. Available at: https://cihr-irsc.gc.ca/e/47332.html [Accessed 30 Aug. 2021].

9. McLean R, Gargani J. Scaling Impact: Innovation for the public good. 2019; London, New York and Ottawa: Routledge and International Development Research Centre. Available at: https://www.idrc.ca/en/book/scaling-impact-innovation-public-good [Accessed 30 Aug. 2021].

10. Mancuso A, Malm SA, Sharkey A, Shahabuddin ASM, Shroff ZC. Cross-cutting lessons from the decision-maker led implementation research initiative. Health Res Policy Sys 2021; 10(Suppl 2): 83 https://doi.org/10.1186/s12961-021-00706-0.

11. Drahota A, Meza RD, Brikho B, Naaf M, Estabillo JA, Gomez ED, Vejnoska SF, Defek S, Stahmer AC, Aarons GA. Community-Academic Partnerships: A Systematic Review of the State of the Literature and Recommendations for Future Research. M Milbank Quarterly. 2016 doi: 10.1111/14680009.12184 .

12. Jull, J, Giles, A, Graham, ID. Community-based Participatory Research and Integrated Knowledge Translation: Advancing the co-creation of knowledge. Implementation Science. 2017; 12:150 https://doi.org/10.1186/s13012-017-0696-3.

13. Greenhalgh, T., Hinton, L., Finlay, T., Macfarlane, A., Fahy, N., Clyde, B. and Chant, A. Frameworks for supporting patient and public involvement in research: Systematic review and co-design pilot. 2019; Health Expectations, 22(4), pp.785-801 
14. Gagliardi AR, Berta W, Kothari A. et al. Integrated knowledge translation (IKT) in health care: a scoping review. Implementation Sci 11, 38 (2015). https://doi.org/10.1186/s13012-016-0399-1

15. Kothari A, Mccutcheon C, Graham ID. Defining Integrated Knowledge Translation and Moving Forward: A Response to Recent Commentaries. Int J Health Policy Manag. 2017 May; 6(5): 299-300. https://10.15171/ijhpm.2017.15

16. Graham I, Tetroe J, Pearson A. Turning Knowledge into Action: Practical Guidance on How to Do Integrated Knowledge Translation. Lippincott-Joanna Briggs Institute Synthesis Science in Healthcare Series. 2014; 21.

17. Nguyen T, Graham ID, Mrklas KJ, Bowen S, Cargo M, Estabrooks CA, Kothari A, Lavis J, Macaulay AC, MacLeod M, Phipps D, Ramsden VR, Renfrew MJ, Salzburg J, Wallerstein N. How does integrated knowledge translation (IKT) compare to other collaborative research approaches to generating and translating knowledge? Learning from experts in the field. Health Research Policy and Systems. 2020; 18 :35 https://doi.org/10.1186/s12961-020-0539-6.

18. Jason LA, Keys CB, Suarez-Balcazar Y, Taylor RR, Davis MI. (Eds.). (2004). Participatory community research: Theories and methods in action. American Psychological

Association. https://doi.org/10.1037/10726-000

19. Integrated Knowledge Translation Research Network site. https://iktrn.ohri.ca/aboutus/what-is-ikt/.

20. Beaulieu M, Breton M, Brousselle A. Conceptualizing 20 years of engaged scholarship: A scoping review. 2018; PLOS ONE 13(2). https://doi.org/10.1371/journal.pone.0193201.

21. Gibbons M, Limoges C, Nowotny H, Schwartzman S, Scott P, Trow M. The New Production of Knowledge: The Dynamics of Science and Research in Contemporary Societies. 1994. London: Sage Publications

22. Mitchell AS. Mode-2 Knowledge Production within Community-Based Sustainability Projects: Applying Textual and Thematic Analytics to Action Research Conversations. Adm. Sci. 2020, https://doi.org/10.3390/admsci10040090.

23. Noel L, Phillips F, Tossas-Milligan K, Spear K, Vanderford NL, Winn RA, Vanderpool RC, Eckhardt SG. Community-Academic Partnerships: Approaches to Engagement. Am Soc Clinical Oncology. 2019. DOI: 10.1200/EDBK_246229

24. Graham ID, Rycroft-Malone J, Kothari A, McCutcheon C. (forthcoming). Research Coproduction. Hoboken, NJ: Wiley \& Sons

25. McLean, RKD., Graham, ID. Carden, F. Research Quality Plus for Co-Production (RQ+ 4 Co-Pro) - A Holistic Approach to Evaluating Co-Production Research. In Graham, ID., Rycroft-Malone J, Kothari, A., Mccutcheon, C. Research Co-Production. (forthcoming). Hoboken, NJ: Wiley \& Sons.

26. McLean RKD, Graham ID, Bosompra K, Choudhry Y, Coen SE, MacLeod M, Manuel C, McCarthy R, Mota A, Peckham D, Tetroe JM, Tucker J. Understanding the performance and impact of public knowledge translation funding interventions: Protocol for an evaluation of Canadian Institutes of Health Research knowledge translation funding programs. 2012; Implementation Science volume 7, Article number: 57 https://DOI.ORG/10.1186/1748-5908-7-57. 
27. Russell J, Fudge N, and Greenhalgh T. The impact of public involvement in health research: what are we measuring? Why are we measuring it? Should we stop measuring it? 2020; Research Involvement and Engagement, 6(1).

28. Greenhalgh T, Fahy N. Research impact in the community-based health sciences: an analysis of 162 case studies from the 2014 UK Research Excellence Framework. 2015; BMC Med 13, 232. https://doi.org/10.1186/s12916-015-0467-4.

29. McLean R., Ofir Z., Etherington E., Acevedo M., Feinstein O. Research Quality Plus (RQ+): Evaluating Research for Global Sustainable Development. 2022. International Development Research Centre. Ottawa. Available at https://www.idrc.ca/en/rqplus.

30. Lebel J, McLean RKD. A better measure of research from the Global South. 2018; Nature, vol. 559, no. 7712, 2018, pp. 23-26) doi: https://doi.org/10.1038/d41586-018-05581-4

31. Kreindler SA. Advancing the evaluation of integrated knowledge translation. Health Research Policy and Systems, 2018. 16(1).

32. Ward M, Schulz AJ, Israel BA, Rice K, Martenies SE, Markarian E. A conceptual framework for evaluating health equity promotion within community-based participatory research partnerships. 2018; Evaluation and Program Planning, 70, pp.25-34.

33. Beckett K, Farr M, Kothari A, Wye L. le May A. Embracing complexity and uncertainty to create impact: exploring the processes and transformative potential of co-produced research through development of a social impact model. 2018; Health Research Policy and Systems, 16(1).

34. Boivin A, L'Espérance A, Gauvin F-P, Dumez V, Macaulay AC, Lehoux P, Abelson J. Patient and public engagement in research and health system decision making: A systematic review of evaluation tools. 2018b; Health Expectations, 21(6).

35. Hsieh HF, Shannon SE. Three approaches to qualitative content analysis. Qual Health Res. 2005;15(9):1277-1288.

36. Phillippi J, Lauderdale J. A Guide to Field Notes for Qualitative Research: Context and Conversation. Qualitative Health Research. 2017. Doi.org/10.1177/1049732317697182.

37. Guest, G., Bunce, A., \& Johnson, L. (2006). How Many Interviews Are Enough?: An Experiment with Data Saturation and Variability. Field Methods, 18(1), 59-

82. https://doi.org/10.1177/1525822X05279903

38. Morgan DL, Ataie J, Carder P, Hoffman K. Introducing dyadic interviews as a method for collecting qualitative data. Qual Health Res. 2013;23(9):1276-1284. doi:10.1177/1049732313501889

39. Nowell, L. S., Norris, J. M., White, D. E., \& Moules, N. J. (2017). Thematic Analysis: Striving to Meet the Trustworthiness Criteria. International Journal of Qualitative Methods, 16(1), 1609406917733847. https://doi.org/10.1177/1609406917733847

\section{Tables}

Table 1. Research co-production traditions 
Tradition Definition

Participatory In participatory research the community is part of shaping the research agenda; Research community members work with researchers on the research itself and on implementation of the agenda [18].

Integrated As defined on the Integrated Knowledge Translation Research Network website, Knowledge 'Integrated Knowledge Translation (IKT) is a model of collaborative research, where Translation researchers work with knowledge users who identify a problem and are in a position to act on the research findings [19].' In short, it is about doing research with the people who use it.

Engaged Similar to the traditions above, Engaged Scholarship engages communities with Scholarship researchers at multiple stages of the research process and focuses on issues that are important to a community. A community may be geographic or a community of interest (e.g., patient engagement in research that affects them) [20].

Mode 2 Research

Mode 2 research is a transdisciplinary approach to research on development problems that engages both researchers and practitioners without strict hierarchy or fixed approaches in the research. The research is co-produced with people who work and live in the domain of the research $[21,22]$.

Community Academic Partnership
Community-academic partnerships optimize the engagement of academic and community resources thereby increasing the pertinence of academic research and trust in findings in the community. Community-academic partnerships support diverse solutions to meet the needs of specific communities [23].
Research

Co-

Production
Research co-production is an umbrella term. The term is used to describe the process of researchers working with research users to create and conduct research together. The aim of research co-production is to bring multiple perspectives into setting research questions and into decision-making about the how the research is done, so that the work reflects the needs of those who will use it [24].

Table 2. Mainstream evaluation stacked against co-production [25] 
Evaluation Challenges for co-production

Approach

\section{Deliberative}

\section{What form}

does it take?

Peer-review at proposal, ethics, publication, and sharing stages of research.
Peer-review relies on researchers, not users or beneficiaries, to judge a proposal or a project in terms of scientific criteria. With few exceptions co-production proposals are assessed by scientific peers, not knowledge users (who are not considered peers). (See for example the work of PCORI (www.PCORI.org) or the former Knowledge Translation Funding Program at CIHR $[8,26]$ for examples of 'Merit Review' in practice.) Further, they use scientific criteria and scientist perspectives to determine whether, 1 ) a study is ethical for participants on behalf of participants (through REB procedures), and, 2) if a study contains publishable results, not actionable results. In our view, scientists' expertise can identify the knowledge gaps the work aims to fill and critique the strength of the methods that will be used to produce it. Yet, without including knowledge users and beneficiaries' significant evaluation gaps persist, as knowledge users are best placed to assess the relevance, significance, utility, and potential impact of the research.

\section{Analytic}

\section{What form} does it take?

Metrics and quantitative indices. For example, bibliometrics, altmetrics, university rankings, journal rankings.
Metrics are biased toward fields of research where productivity in creating output is paramount, largely, the scholarly paper published in a peer-reviewed, indexed journal. They are also biased toward the quantification of outputs. Metrics and their aggregations tell us little, if anything, about the quality of the engagement of users in a project. Neither do they speak to the policy or practice relevance of a research topic, or the actual implications of the work for intended beneficiaries. Moreover, they are largely blind to research results that fall outside the indices of mainstream, English-language, academic journal publishing. Similarly, real-world impact resulting from co-production typically goes uncounted with the analytic paradigm.

\section{Research impact assessment (RIA)}

\section{What form} does it take?

Retrospective reviews, often case studies with
For co-producers whose aim is knowledge uptake and use, the RIA approach seems welcome at first glance. In some cases, the RIA may even privilege research co-production which can be well positioned to accelerate the uptake and impact of research by knowledge-users. However, RIA is not a complete solution for research co-production quality evaluation. RIA may provide a meaningful measure for funders and organizations whose primary concern is amplifying or modifying the magnitude of impact they can demonstrate and communicate; additionally, it does not systematically recognize and study the process of user-engagement and how it can set a course and even create social change during study design and implementation $[27,28]$. Furthermore, the mismatch between research funding trajectories (typically $1-5$ years) and research impact trajectories (typically $10-20$ years) leaves a significant gap in our knowledge of how to do better co-production. 
social and

economic

measures.

Table 3. What $R Q+4$ Co-Pro can learn, benefit from, and build on from existing frameworks, experiences, and systematic reviews [25] 

Article/
Recommendation
Framework (from the paper cited)
\&
Theoretical
Lens

Lesson for $R Q+4$ Co-Pro

Integrated

Knowledge

Translation

[31]
(IKT) lens

Accept context as inseparable component of a causal chain

Use a realist evaluation approach to highlight context in evaluations of IKT foreground of evaluations and
Communitybased

Participatory

Research

$(C B P R)$ lens

[32]

Co-

Production

lens [33]

Equity is critical to understanding CBPR process \& impact for communities, and thus, should be in the informed by various methods and instruments

Recognize social and transformational effects of coproduction, including both those that occur as a part of the research process (as a result of productive interactions) and those related to research results

Patient \& Public Engagement lens [34]
Increase scientific rigour of framework development
Use the three Contextual Factors of the framework to categorize and study context-mechanism interactions
$R Q+4$ Co-Pro identifies Contextual Factors as a framework component

$R Q+4$ Co-Pro names Sub-Dimensions that prioritize and critically interrogate equity: 2.1. Inclusion of Local Knowledge \& Ways of Knowing; 2.2. Trust, Power \& Mutually Beneficial Partnerships; 2.3 . Intersectionality 2.4 . Negative Consequences

$R Q+4$ Co-Pro sheds light on the process of engagement and utility of results, by naming both elements in specific Quality Dimensions of the framework ( 2 \& 3 ), and by doing so, highlights 'the hidden' relational and at times transformational benefits of co-production
Include stakeholders in framework development

Improve accessibility of frameworks (understandability/readability)
$R Q+4$ Co-Pro is derived from the validity and reliability tested, theory informed, $\mathrm{RQ}+$ approach and framework of the International Development Research Centre

$\mathrm{RQ}+4$ Co-Pro will be field tested in the study described herein, which is structured as a stakeholder inclusive IKT effort.
Patient \& Public Involvement lens [13]
A single, one-sized fits all framework is unlikely to emerge. Instead, co-develop
Ensure simple, clear, accessible publications in various formats and a well-developed sharing strategy. This publication is one component. As results of this field test emerge, sharing and use strategies will be developed with/by the KUs on the study team.

$\mathrm{RQ}+4$ Co-Pro is a modular construct, whereby new users can adapt and re-shape the framework components to match their values and objectives, while keeping intact the three tenets that address 
frameworks for local contexts, principles, and objectives. the shortcomings of status quo deliberative, analytic, and RIA evaluation approaches when applied to co-production.
Public Involvement lens [26]
Acknowledge: 1) the different rationales for public involvement, 2) that there may be negative impacts, 3 ) the role of power relations
$\mathrm{RQ}+4$ Co-Pro has specific Sub-Dimensions (2.1, $2.2,2.3,2.4)$ that prioritize and will help the field to learn about power dynamics and potentially negative consequences of co-production.

Table 4. Project eligibility criteria

Inclusion criteria

$\bigotimes \quad$ The research project uses Integrated Knowledge Translation.

Q The project is led by a member of the IKT Research Network

\ The project is either complete or near completion (i.e., has draft products)

$\quad$ The project prioritizes health system actors as knowledge users

\section{Exclusion criteria}

Q Projects focused on science of IKT

$\nabla \quad$ Projects focused on training and curriculum development for IKT

Table 5. Participant eligibility criteria

Inclusion criteria

$\bigotimes \quad$ Individual must have been a member of the IKT Research Network

$\Downarrow \quad$ Individual must be a member of the IKT Research Network

\ Individual willing to both submit a project for assessment and act as an assessor in a dyad with another project
Exclusion criteria

Q Individual not able to participate in English

\section{Figures}




\section{Key definitions:}

Research Quality Plus (RQ+) is an approach to assessing the quality of research which focuses on problem solving. It uses both qualitative and quantitative methods and may be used at the design, delivery and dissemination stages of the research to assess whether the research has been designed, delivered, and communicated in ways that enhance its potential for use $[29,30]$.

Research Quality Plus for Co-Production (RQ+ 4 Co-Pro) is an approach to assessing the quality of research co-production. It prioritizes the importance of the knowledge user's involvement in the research design, research conduct and implementation of the research findings for high-quality coproduction research (25).

\section{Figure 1}

Key definitions 
$\mathrm{RO}+4 \mathrm{Co}-\mathrm{Pro}$ is an approach to defining and evaluating the quality of coproduction. It allows tailoring to context, values, and purpose. It can

support planning, management and learning across the lifecycle of a coproduction project, program or organization.

\section{The RQ+ 4 Co-Pro Framework}

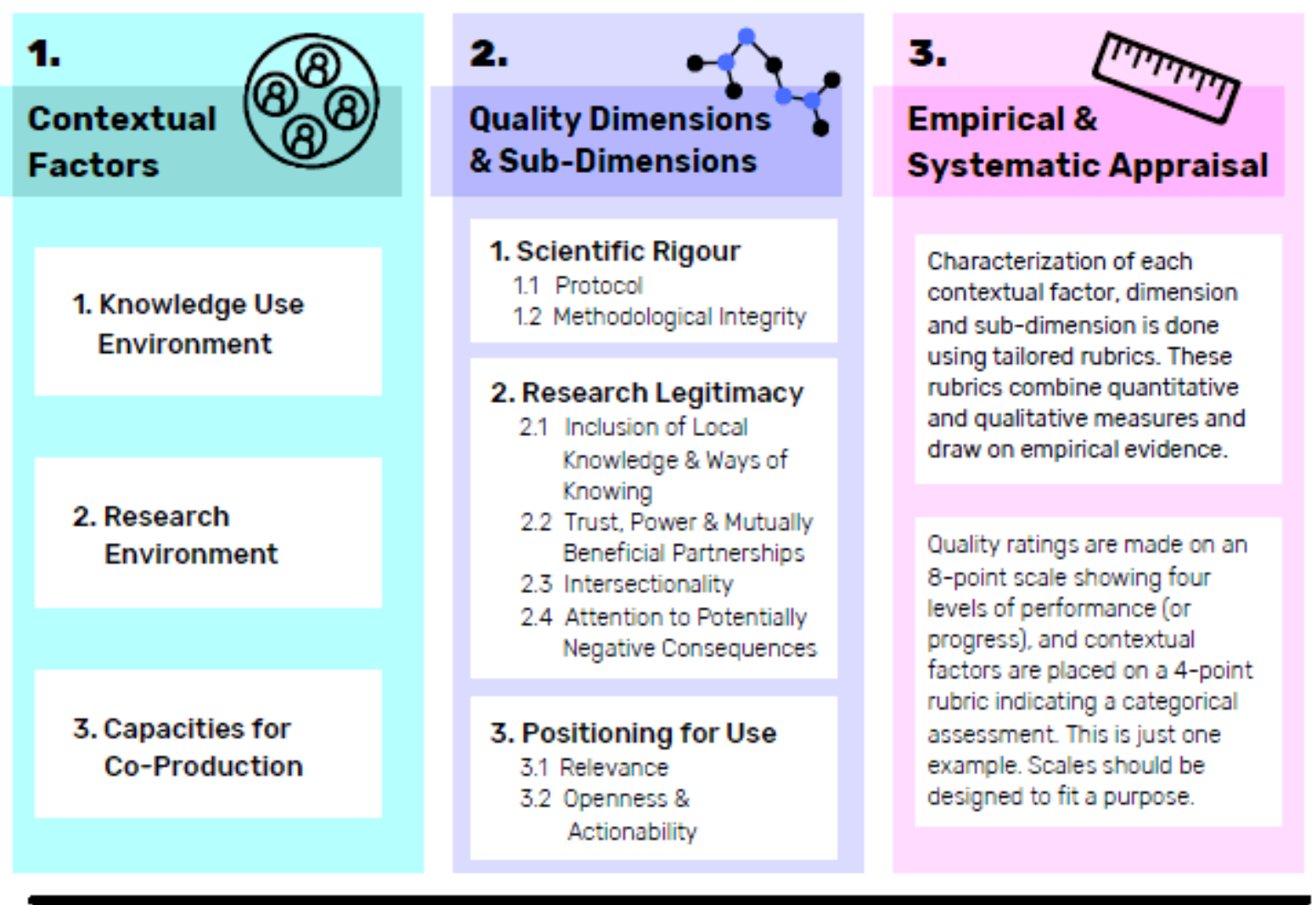

Authors: Robert McLean, Ian Graham, Fred Carden

Figure 2

The RQ+ 4 Co-Pro Framework [adapted from infographic originally published by authors (RKDM, IDG, FC), and secondly in 25] 

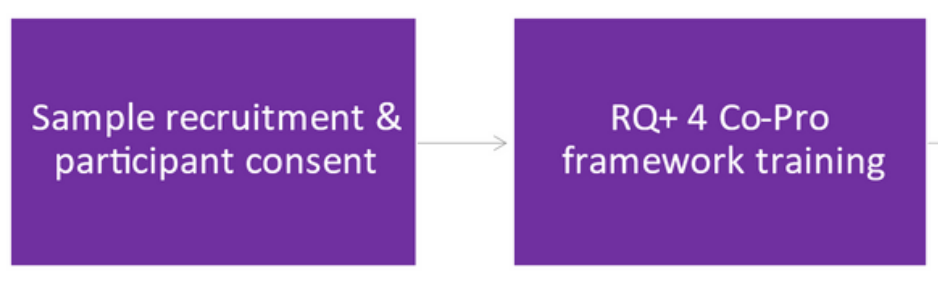

Participant Socio-

Demographic Form \&

Project Information Form

(data collection steps 1\&2)

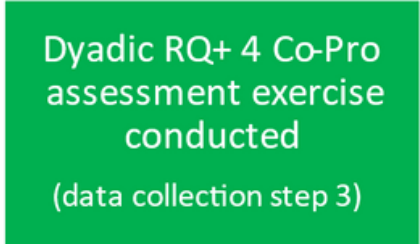
Qualitative interviews with study participants
(data collection step 4)

\section{Data Analysis}

(data analysis steps 1-4)

Revision of the $\mathrm{RQ}+4$ Co-Pro framework
Publication of trial

results, andtested

$\mathrm{RQ}+4$ Co-Pro

framework
Study preparation

Data collection

Analysis \& revision

Sharing results

\section{Figure 3}

Outline of the research life cycle

\section{Supplementary Files}

This is a list of supplementary files associated with this preprint. Click to download.

- Appendix1RQ4CoProFieldTestAssessmentTemplate.docx

- Appendix2CrosswalkofRQwithRQ4CoPro.docx 